\title{
Improving LTE Femtocell Base Station Network Performance by Distributed Power Control
}

\author{
Hwang-Cheng Wang ${ }^{1}$, Fang-Chang Kuo ${ }^{1}$, Chih-Cheng Tseng ${ }^{1, *}$, Bo-Wei Wang ${ }^{1}$, Kuo-Chang Ting ${ }^{2,3}$ \\ ${ }^{1}$ Department of Electronic Engineering, National Ilan University, Taiwan \\ ${ }^{2}$ Department of Business Administration, Minghsin University of Science and Technology, Taiwan \\ ${ }^{3}$ Department of Computer Science and Information Engineering, Minghsin University of Science and Technology, Taiwan
}

Copyright $\odot 2016$ by authors, all rights reserved. Authors agree that this article remains permanently open access under the terms of the Creative Commons Attribution License 4.0 International License

\begin{abstract}
LTE femtocell base stations (FBSs) have expanded the coverage of wireless communication systems. The growing use of FBSs has brought a serious issue of inter-FBS interference (also referred to as co-tier interference) due to their easy and convenient installation. This study proposes a systematic approach to reduce FBS co-tier downlink interference. In this study, FBSs are densely deployed in an environment and femtocell user equipment (FUEs) detect interference from FBSs and send an alarm signal to each FBS which interferes with it. To minimize the negative effects of interference, power levels are reduced for FBSs identified as excessive interference sources, which are defined as FBSs that have received a number of alarm signals over a given threshold. The method proposed in this study has been validated through simulation and is found to effectively reduce co-tier downlink interference in shared-spectrum femtocell environments, thereby improving system performance.
\end{abstract}

Keywords Femtocell, Co-tier Downlink Interference, Alarm Signal, FBS-excessive Interference Source, Power Control

\section{Introduction}

In recent years, global mobile data traffic has grown eleven-fold [1], urging telecommunications carriers to increase network capacity. Frequency can be effectively increased by using a larger amount of small cellular base stations. For this reason, LTE femtocell base stations and related technologies have developed rapidly. Due to higher flexibility and coordination of wireless communication, the quality of indoor cellular signals has improved and the number of outdoor users of large base stations has increased. Moreover, the high density of base stations and mobile devices is a feature of $5 \mathrm{G}$ mobile networks [2]. However, the growing use of femtocell base stations (FBSs) has brought a serious issue of inter-FBS interference (also referred to as co-tier interference) due to their easy and convenient installation. This study examined methods to reduce FBS co-tier downlink interference.

Cognitive radio (CR) [3] has become a powerful tool to improve spectrum efficiency and allows the coexistence of heterogeneous wireless networks in the same area and in the same frequency band. CR devices possess the ability to monitor the environment and inform resource allocation controller of the quality of available spectrum. Therefore, the resource allocation controller may dynamically select available channels and use appropriate transmission parameters in order to avoid harmful interference among competing users. The main features of $\mathrm{CR}$ include spectrum detection, dynamic resource allocation, spectrum sharing and migration.

Another mechanism for utilizing idle frequency band is small cell group muting (SCGM) method. Small cell is a generic term to refer to the various types of scaled-down base stations to extend radio coverage or enhance radio channel quality. An FBS can be considered a special type of small cell. Enhanced cell interference coordination (eICIC) and time-domain masking technology have been extensively studied and are now part of the LTE-A specification. In [4], a practical small cell network (SCN) architecture has been proposed. Specifically, the proposed method can eliminate interference between macro cell and small cells to achieve low power consumption. Under the above SCN architecture, a small cell group muting scheme was also proposed to reduce interference between adjacent small cells. The effectiveness of SCN architecture and SCGM has been verified through simulation results.

In this study, FBSs are assumed to provide resources over a shared spectrum and operate in an open subscriber group (OSG) mode [5]. Randomly deployed and turned on and off by users, FBSs are characterized by a high level of randomness. Studies on cognitive radio [6][7] proposed to use femtocell user equipment (FUE) for algorithm design. In this study, FBS planning is assumed to undergo distributed management [8] rather than data collection and exchange via 
a femtocell management system (FMS) [9]. The number of alarms received by interference sources from FUE is the main factor examined in this study and is used to control data exchange. Matlab simulation results indicated that the proposed model can effectively reduce co-tier downlink interference in shared-spectrum femtocell environments.

\section{System Model}

Femtocells are assumed to be deployed over the area covered by one macro base station (MBS). This study only considers co-tier downlink interference; i.e., interference resulting from FBSs that use the same downlink frequency band for communication with FUEs.

Assume $M$ FBSs are deployed, and $N$ FUEs operating within the area are served by the FBSs. Each FUE or FBS is equipped with only one antenna. In this study, cross-tier interference of MBS to FBSs is ignored and only co-tier interference is examined. FBSs and FUEs are randomly distributed throughout the simulation environment, with one FBS providing service to at most 10 FUEs and each FUE receiving service from exactly one FBS.

The FBSs are not controlled by an FMS. FUEs are affected by co-tier interference if they are located within the coverage of non-serving FBSs. When an FUE detects interference [10], it broadcasts an alarm which is received by FBSs within range. Thus, each FBS obtains information about which and how many FUEs are affected by it at any time [11][12]. Interfering FBSs are identified as FBS-EI sources if the number of alarms received reaches the threshold. Such FBSs require reduction of their power level.

The transmission of alarms is illustrated in Figure 1. For clarity, in this and subsequent figures, FUEs served by a particular FBS, say, FBS- $j$ are denoted by FBS- $j_{-} 1$, FBS- $j_{-} 2$, and so on. It can be seen that several FBSs have overlapping coverage areas. For instance, FBSs 1 to 4 all overlap with FBS-5 in radio coverage. FUEs located inside the overlapping areas are thus affected by co-tier interference.

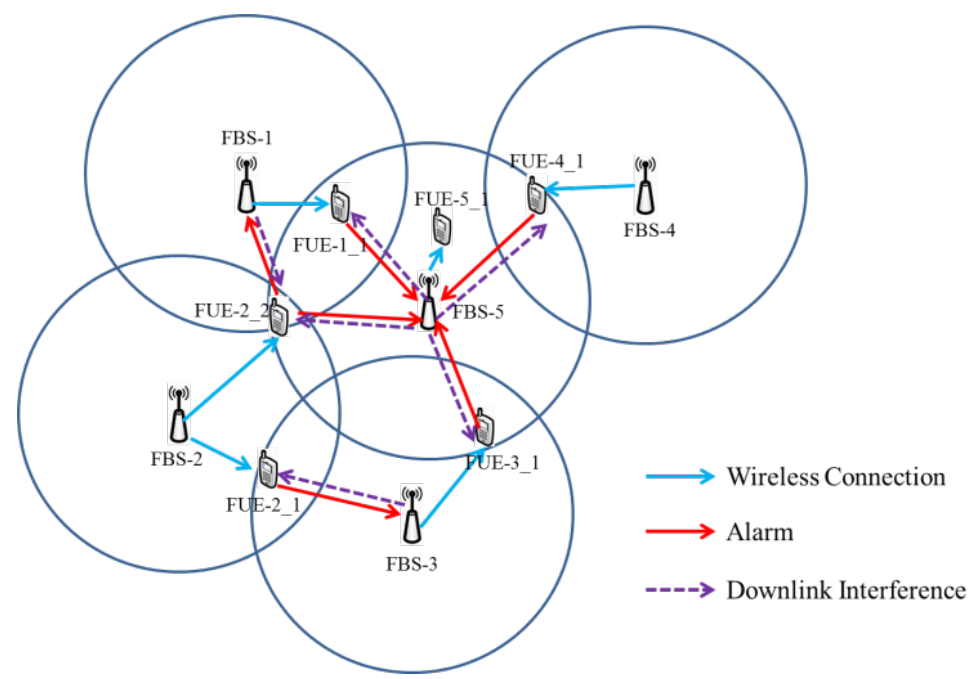

Figure 1. Schematic illustration of alarm signaling

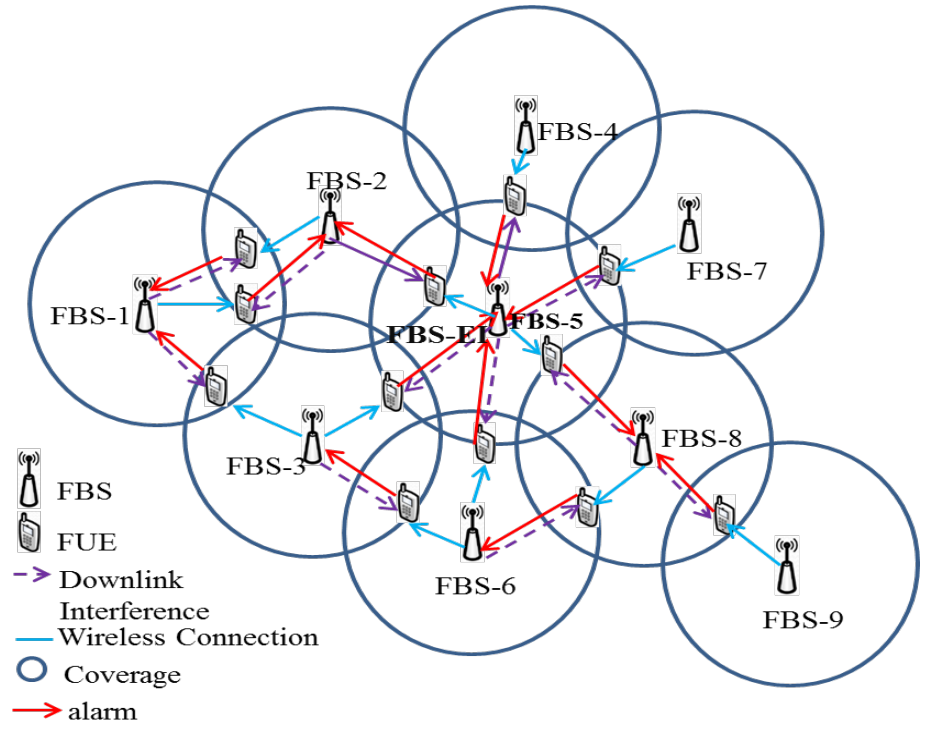

Figure 2. Coverage of FBSs with no power control 


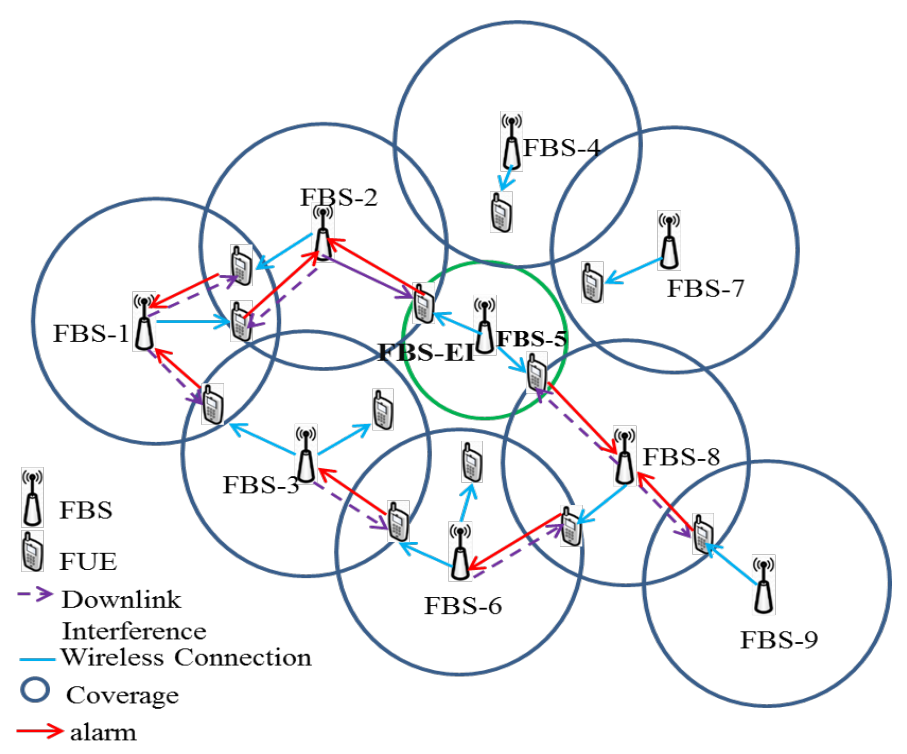

Figure 3. Coverage of FBS-EI source (FBS-5) after power control is performed.

FBSs receiving many alarms are expected to cause interference for many FUEs. The interference levels in the environment can be decreased and the overall efficiency can be improved by controlling the power in such FBSs. FBSs for which the number of alarms reached the threshold (which will be discussed later) are defined as FBS-EI sources. Thus, the number of alarms determines whether FBSs required power control. Figure 2 shows a system in which no power adjustment is made.

The transmit power of FBSs and FUEs is equal and FUEs can sense which FBSs cause interference. Each FUE is able to detect interference from surrounding FBSs. Those FBSs that are located within the radio range of an FUE will receive alarm signals from it due to limitation of antenna sensitivity. If the number of alarms received by an FBS is higher than a specified threshold, the FBS is identified as an FBS-EI source and is required to perform power control. No changes are made to the transmit power level of those FBSs for which the number of received alarm signals is under the threshold.

As seen in Figure 3, many FUEs are interfered by FBS-5; therefore, the transmit power of FBS-5 is reduced. This considerably reduces the interference to FUEs served by the FBSs 3, 4, 6, and 7. At the same time, FUEs under FBS-5 continue to operate with weaker signals due to the reduction in transmit power of FBS-5.

\section{Problem Formulation}

As mentioned previously, FBS-EI sources are considered problematic FBSs as they interfere with a multitude of FUEs. After FBS-EI sources are identified, interference can be reduced through power control or frequency reuse. In this study, power control is applied to FBS-EI sources, thus reducing their communication range. FBSs are determined as FBS-EI sources if the number of alarms they receive is over a threshold value $T$. The optimal value of $T$ is obtained by simulation. In the subsequent analysis, the following assumptions are made:

- Each FUE is connected to the nearest FBS.

- Each FUE can sense interference from FBSs located within its range.

- Each FUE transmits an alarm signal to sources of interference located within its range.

We propose the interference-aware power control (IAPC) scheme. To facilitate the description of the scheme, we introduce the following notations:

- $\quad P_{j}$ : actual transmit power of FBS- $i$.

- $P_{n p c}$ : transmit power of FBS with no power control (NPC).

- $R S S_{i, i_{-}}$: the signal strength received by FUE-i_l from FBS- $i$.

- $\quad I_{j, i} l_{l}$ : the interference suffered by FUE- $i_{-} l$ from FBS- $j$.

- $R_{\text {min }}$ : minimum RSS level for each FUE. To allow communication with FBS-j, $R S S_{j, j k}$ of the signal received by FUE-j $j k$ from FBS- $j$ must be at least $R_{\text {min. }}$.

- $\quad \mathbb{F}_{j}$ : the set of FUEs serviced by FBS- $j$.

- $L_{i, j}$ : path loss from FBS- $i$ to FUE-j_k $k$.

- $\quad C_{i}$ i: capacity of FUE- $i$ l $l$.

- $\quad \eta$ : thermal noise power. 


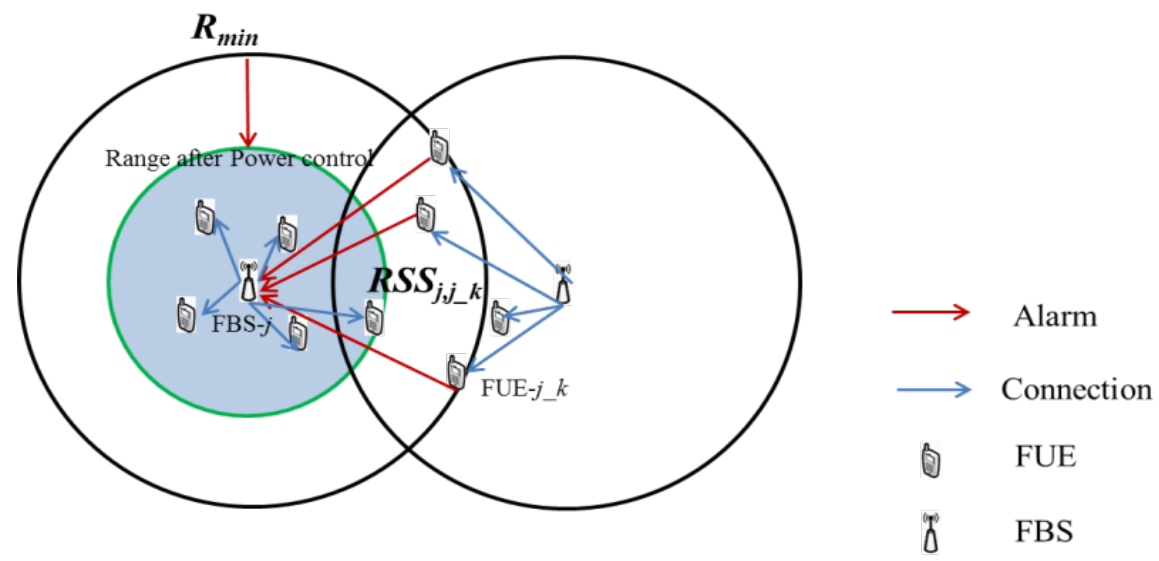

Figure 4. Method of power control for an FBS-EI source

An FBS-EI source requiring power control is shown in Figure 4. Its transmit power is reduced to the minimum level sufficient for providing service to an FUE with the lowest RSS.

Figure 4 gives the scenario before and after power control. For successful communication with the FBS, the strength of signals received by FUE- $j$ located within both the original FBS range (black circle) and power-constrained FBS range (green circle) should be at least $R_{\text {min }}$.

As seen from Figure 4, at first FBS- $j$ uses power $P_{j}$ to transmit signals under no power control (NPC) condition. The received signal strength (RSS) of FUE- $j_{-} k$ after suffering path loss $L_{j, j k}$ is:

$$
P_{j}-L_{j, j_{-} k}=R S S_{j, j_{-} k}
$$

If no power control is performed, the transmit power of FBS- $j$ is $P_{j}=P_{n p c}$. Thus, we have

$$
\begin{aligned}
& P_{n p c}-L_{j, j_{-} k}=R S S_{j, j_{-} k}, \\
& \text { for } j=1,2, \ldots, M ; k=1,2, \ldots,\left|\mathbb{F}_{j}\right|
\end{aligned}
$$

If power control is performed, $R S S_{j, j \in k}$ of the signal received by FUE- $j \_k$ is equal to $R_{\min }$. Hence,

$$
R_{\min }=P_{j}-L_{j, j_{-} k}
$$

The transmit power for each FBS after applying the IAPC algorithm can be calculated using equations (6) and (7). Given the strength of the signal FUE- $j_{-} k$ receives from FBS- $j, P_{j}$ can be calculated as follows:

$$
P_{j}=P_{n p c}+\left(R_{\min }-\min _{j_{-} k \in \mathbb{F}_{j}} R S S_{j, j_{-} k}\right)
$$

Eq. (8) ensures that after the reduction in the communication range of FBS-EI according to the IAPC algorithm, the service provided to FUEs by FBS-EI will not be compromised.

Suppose FUE- $i$ - $l$ is subject to downlink interference from other non-serving FBSs. The total interference can be expressed as

$$
I_{i_{-} l}=\sum_{j=1, j \neq i}^{M} I_{j, i_{-} l}
$$

where $I_{j, i_{-} l}=P_{j}-L_{j, i_{l} l}$ is the interference of FBS- $j$ to FUE- $i l_{-} l$, with $P_{j}$ the transmit power of FBS- $j$ and $L_{j, i} i_{l}$ the path loss between FBS- $j$ and FUE- $i$ l $l$.

The signal strength received by FUE- $i_{-} l$ from FBS- $i$ and experiencing path loss $L_{i, i_{-} l}$ has the strength of $R S S_{i_{-} l}$ which can be written as

$$
R S S_{i_{-} l}=P_{i} / L_{i, i_{-} l}
$$

The SINR perceived by FUE- $i$ - $l$ can be obtained as

$$
\operatorname{SINR}_{i_{-} l}=R S S_{i_{-} l} /\left(I_{i_{-} l}+\eta\right)
$$

The path loss model used in the simulation is specified in 3GPP TR25.952 [13] :

$$
L_{i, j_{-} k}(\mathrm{~dB})=37+30 \log _{10}\left(D_{i, j_{-} k}\right)
$$

where $D_{i, j_{-} k}$ is the distance between FBS- $i$ and FUE- $j_{-} k$ in meters. As $D_{i, j \_}$increases, so does the path loss $L_{i, j_{-} k}$. The Shannon formula of capacity [14] is referred to when computing the capacity:

$$
C_{i_{-} l}=\left(B /\left|\mathbb{F}_{i}\right|\right) \log _{2}\left(1+\operatorname{SINR}_{i_{-} l}\right)
$$

where $B$ is the system bandwidth.

\section{Simulation and Discussion}

\section{Simulation setup}

It was found that the optimal capacity of the environment can be achieved when $T=2$ after a series of simulations. This section compares NPC and IAPC and examines the growth of the overall capacity for $T=2$. RSS, interference, SINR, and capacity are recorded and used as the performance metrics. Parameters of the simulation environments are given in Table 1. 
Table 1. Simulation parameters

\begin{tabular}{|c|c|}
\hline Parameter & Value \\
\hline Map Range & $200 \mathrm{~m} \times 200 \mathrm{~m}$ \\
\hline Number of FBSs (M) & 100 \\
\hline Number of FUEs (N) & 300 \\
\hline FBS Radius of Coverage & $20 \mathrm{~m}$ \\
\hline FBS Transmit Power & $20 \mathrm{dBm}$ \\
\hline Bandwidth & $10 \mathrm{MHz}$ \\
\hline Frequency & $2 \mathrm{GHz}$ \\
\hline Rmin & $-56 \mathrm{dBm}$ \\
\hline
\end{tabular}

Ten rounds of simulation were conducted using the parameters in Table 1 but with different deployed distributions of FBS and FUEs. The mean values were calculated for each round of simulation. The different distribution used in each round is to confirm that the environment can be improved regardless of the distribution of FBSs and FUEs. Performance results for each round are the arithmetic mean values of the results collected from all FUEs.

\section{Simulation Results and Discussion}

Figure 5 shows the deployment of FBSs and FUEs in one round. Red circles represent FUEs. After the first stage, the FBSs are classified as FBS-EI sources and non-FBS-EI sources, and shown in green and blue squares, respectively. As can be seen, in areas of dense FBS deployment there are lots of green squares due to mutual interference on FUEs serviced by different FBSs. Based on the results, 21 FBSs belong to the latter category, while the rest are FBS-EI sources.

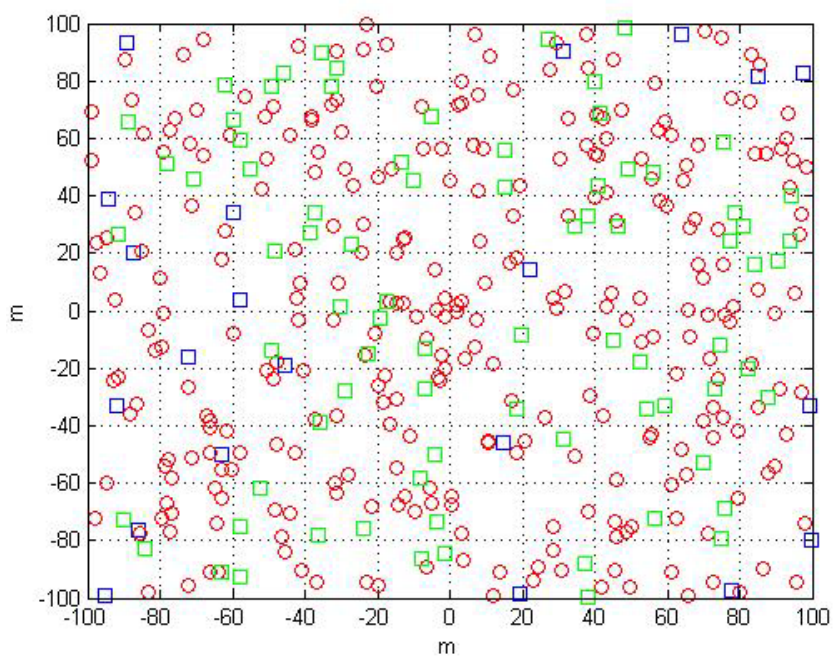

Figure 5. Deployment of FBSs and FUEs in one round of simulation

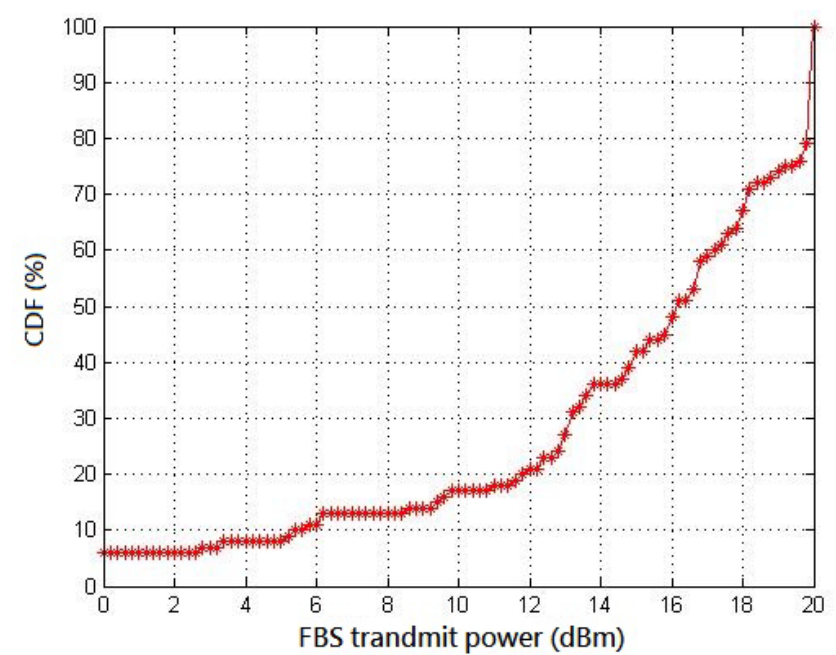

Figure 6. Distribution of FBS transmit power after power control on FBS-EI sources.

Figure 6 depicts the distribution of the transmit power of FBSs after power control is performed on FBS-EI sources. Out of the $100 \mathrm{FBSs}, 12$ have transmit power lower than 10 $\mathrm{dBm}, 25$ fall in the range $[10,15] \mathrm{dBm}$, and the rest are above $15 \mathrm{dBm}$. Note that several FBSs have a transmit power of $0 \mathrm{dBm}$. This is because we assume that if an FBS does not serve any FUE but interfere with other FUEs, its power will be set to a minimum value of $0 \mathrm{dBm}$. Based on our way of power control, the majority of FBS-EI sources maintain a transmit power of over $15 \mathrm{dBm}$.

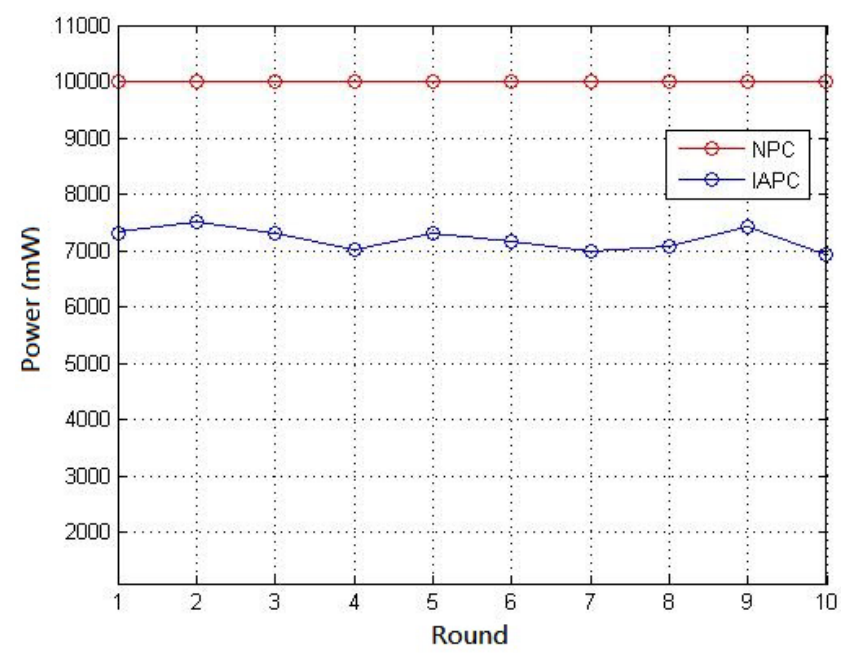

Figure 7. Power consumption with and without power control

Figure 7 demonstrates the total power consumption of the $100 \mathrm{FBSs}$ in each round with no power control and with power control based on the IAPC algorithm. It shows that with no power control, the overall power consumption is fixed at $10000 \mathrm{~mW}$. On the other hand, when power control is performed, the power consumption by all the FBSs is lowered to 7000 7500 $\mathrm{mW}$. The results reflect the fact that IAPC can not only mitigate interference but also conserve energy. 


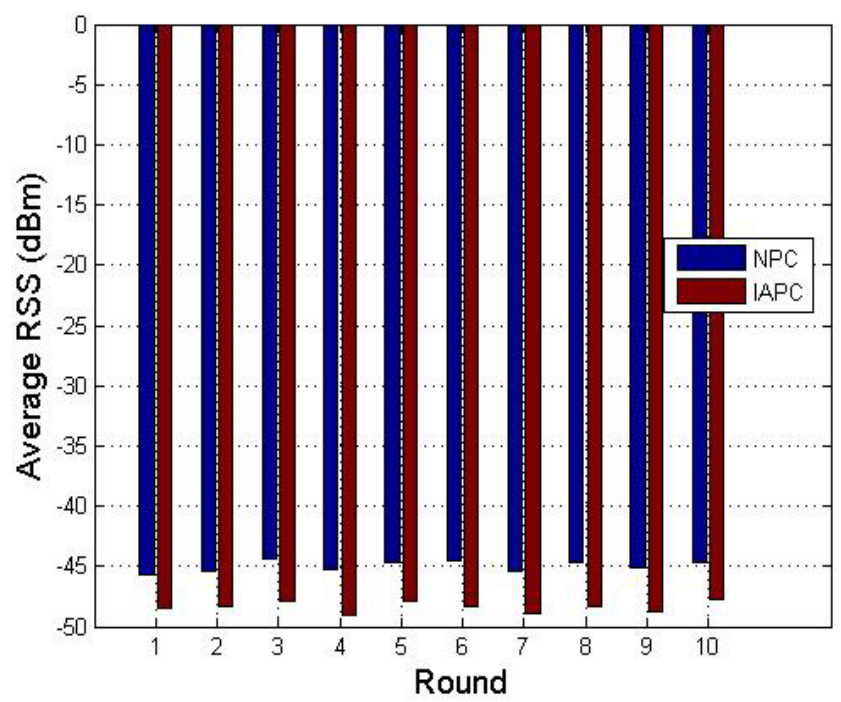

Figure 8. RSS for IAPC and NPC

The strength of received signals under NPC was found to be better than that under IAPC, with a small difference between the two (approximately $4 \mathrm{~dB}$ ) (Figure 8), indicating that, apart from reducing power consumption, power control has a slight effect on the signal strength.

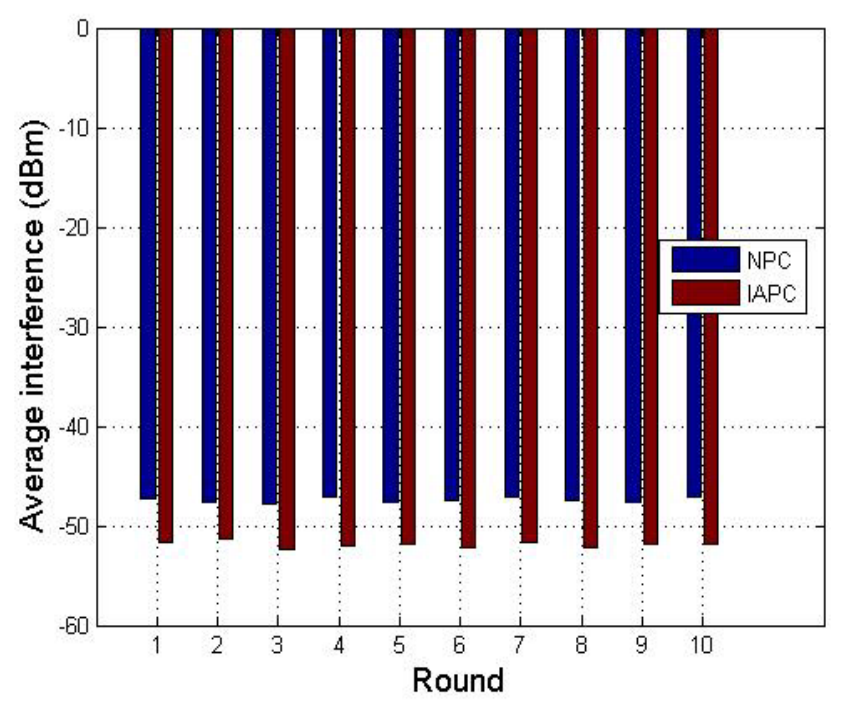

Figure 9. Interference for IAPC and NPC

Interference under IAPC was found to be smaller than that under NPC (Figure 9). Interference was considerably reduced, although at the expense of weakened signal strength. The results of ten simulation rounds demonstrate that the proposed approach can effectively reduce signal interference independent of FBS and FUE locations. Comparison of the SINR under NPC and IAPC conditions is depicted in Figure 10. Despite the decline in signal strength resulting from power reduction, IAPC improves SINR by 1 to $1.5 \mathrm{~dB}$ by providing an environment with less interference.

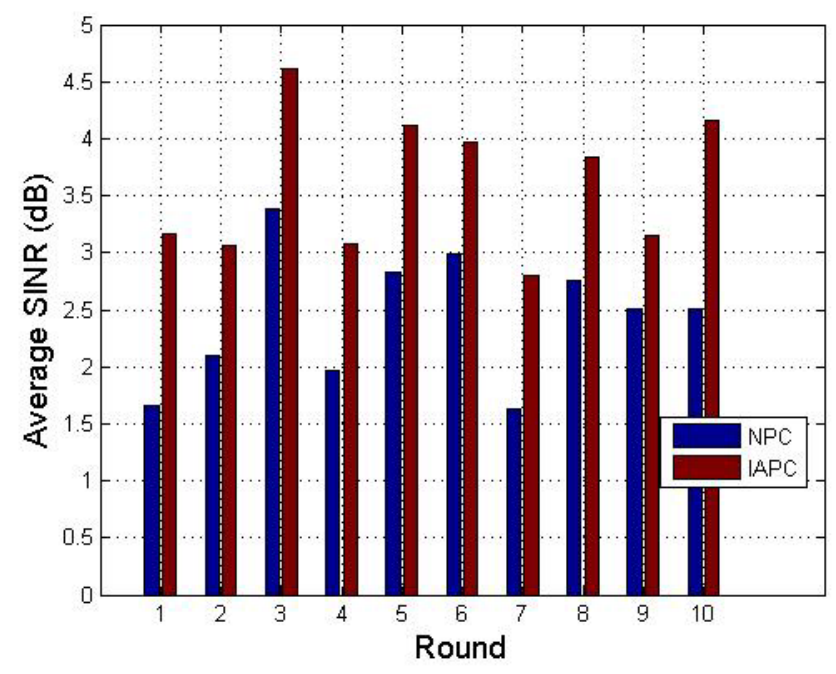

Figure 10. SINR for IAPC and NPC

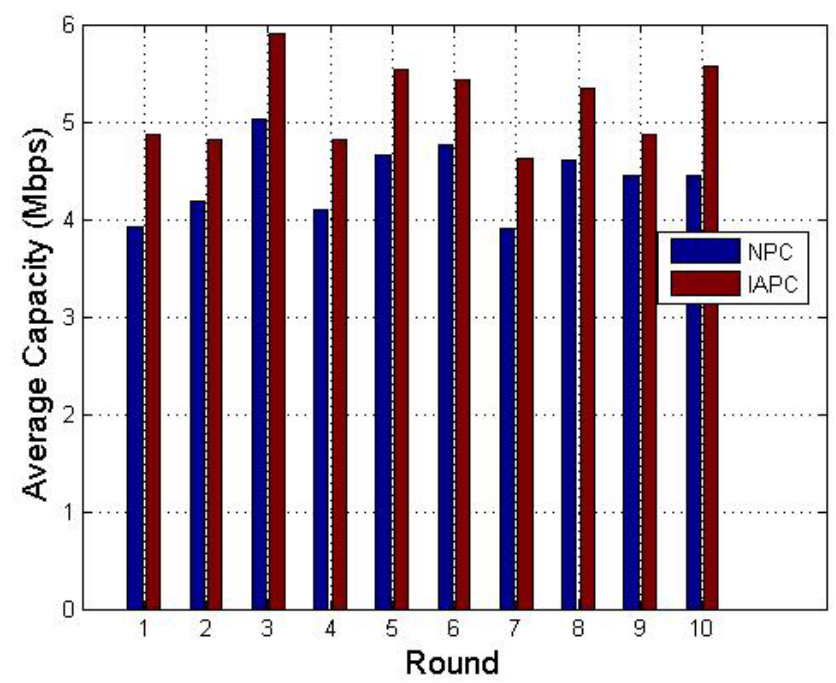

Figure 11. Capacity for IAPC and NPC

In addition to the increased SINR, IAPC improves the average capacity of each FUE in each simulation round by approximately $20 \%$ (Figure 11 ). The proposed scheme can thus provide improved capacity independent of the deployment of FBSs and FUEs.

\section{Concluding Remarks}

Cross- and co-tier interference can occur in shared-spectrum femtocell environments. This study investigated inter-FBS downlink interference and proposed a method to reduce interference and increase network capacity via FBS power control. One salient feature of the proposed method is the distributed approach for finding FBS-EI sources. Depending on the number of alarms received by base stations from FUEs, FBS-EI sources are identified. This greatly reduces the number of information exchanges among FBSs. The IAPC algorithm is then applied to control the transmit power of FBS-EI sources. Simulation results 
validated the effectiveness of IAPC as it was shown to improve network efficiency regardless of FUE locations. Comparison of system performance before and after IAPC was applied showed that the proposed approach reduced interference to users and improved system performance. Moreover, IAPC helps reduce overall power consumption via power control. Although the reduction in power consumption is not the highest priority for FBSs, it can cut down expenditures and increase the willingness of users to adopt femtocells.

\section{Acknowledgements}

This research was supported by Ministry of Science and Technology, Taiwan, ROC, under grant numbers 104-2221-E-197-007, 104-2221-E-197-009, and 104-2221-E-197-016.

\section{REFERENCES}

[1] http://www.smallcellforum.org/about/about-small-cells/elevat or-pitch/

[2] J. G. Andrews, S. Buzzi, W. Choi, S. V. Hanly, A. Lozano, A C. K. Soong, and J. C. Zhang, "What will $5 \mathrm{G}$ be? " IEEE Journal on Selected Areas in Communications, vol. 32, no. 6, pp. 1065-1082, June 2014.

[3] L. Zhang, L. Yang, and T. Yang, "Cognitive interference management for LTE-A femtocells with distributed carrier selection," IEEE 72nd VTC 2010-Fall, pp.1-5, September 2010.

[4] Y. P. Zhang, S. Feng, P. Zhang, L. Xia, Y. C. Wu, and X. Ren, "Inter-cell interference management in LTE-A small-cell networks," IEEE 77th VTC-Spring, pp. 1-6, June 2013.

[5] V. Sathya, H. V. Gudivada, H. Narayanam, B. M. Krishna, and B. R. Tamma, "Enhanced distributed resource allocation and interference management in LTE femtocell networks," IEEE
International Conference on Wireless and Mobile Computing, Networking and Communications, pp. 553-558, October 2013.

[6] J. Jin and B. Li, "Cooperative resource management in cognitive WiMAX with femtocells," The 29th Conference on Information Communications (INFOCOM 2010), San Diego, CA, USA, pp. 1-9, March 2010.

[7] A. Attar, V. Krishnamurthy, and O. N. Gharehshiran, "Interference management using cognitive base-stations for UMTS LTE," IEEE Communications Magazine, vol. 49, no. 8, pp. 152-159, 2011.

[8] E. Hossain, "Evolution toward 5G cellular networks: a radio resource and interference management perspective," Tutorial, IEEE ICC 2014, June 2014.

[9] Y. S. Liang, W. H. Chung, G. K. Ni, I. Y. Chen, H. Zhang, and S. Y. Kuo, "Resource allocation with interference avoidance in OFDMA femtocell network," IEEE Trans. Vehicular Technology, vol. 61, no. 5, pp. 2243-2255, June 2012.

[10] A. De Domenico, E. C. Strinati, and M. G. Di Benedetto, "Cognitive strategies for green two-tier cellular networks: a critical overview," in M. S. Obaidat, A. Anpalagan, and I. Woungang (eds.), Handbook of Green Information and Communication Systems, chap. 1, Academic Press, 2013.

[11] E. B. Rodrigues, "Optimal distributed frequency planning for OFDMA femtocell networks," IEEE 9th International Conference on Personal Indoor and Mobile Radio Communications (PIMRC), pp. 2914-2918, September 2013.

[12] V. Sathya, H. V. Gudivada, H. Narayanam, B. M. Krishna, and B. R. Tamma, "Enhanced distributed resource allocation and interference management in LTE femtocell networks," IEEE International Conference on Wireless and Mobile Computing, Networking and Communications, pp. 553-558, October 2013.

[13] 3rd Generation Partnership Project; Technical Specification Group Radio Access Network; TDD Base Station Classification (Release 2000), ftp://www.3gpp.org/tsg_ran/T SG_RAN/TSGR_12/Docs/PDFs/RP-010457.pdf.

[14] V. Chandrasekhar and J. G. Andrews, "Spectrum allocation in two-tier networks," IEEE Trans. Commun., vol. 57, no. 10, pp. 3059-3068, Oct. 2009. 